\title{
Numerically Efficient Analysis of Planar Microstrip Configurations Using Closed-Form Green's Functions
}

\author{
Ikmo Park, Raj Mittra, Fellow, IEEE, and M. I. Aksun
}

\begin{abstract}
An efficient technique for the analysis of a general class of microstrip structures with a substrate and a superstrate is investigated in this paper using newly-derived closed-form spatial domain Green's functions employed in conjunction with the Method of Moments (MoM). The computed current distributions on the microstrip structure are used to determine the scattering parameters of microstrip discontinuities and the input impedances of microstrip patch antennas. It is shown that the use of the closed-form Green's functions in the context of the MoM provides a computational advantage in terms of the CPU time by almost two orders of magnitude over the conventional spectral domain approach employing the transformed version of the Green's functions.
\end{abstract}

\section{INTRODUCTION}

$\mathbf{R}$ ECENT advances in packaging technology of microwave and millimeter wave integrated circuits (MMICs) have engendered a considerable amount of interest in the development of computer-aided design tools for these packages. A variety of approximate techniques, e.g., the quasi-static methods [1]-[3], equivalent waveguide models [4], [5], and segmentation approaches [6], [7], have been employed for the purpose of analyzing MMIC circuits. Although numerically efficient, these techniques do not always provide results that are sufficiently accurate. An alternative is to use a more sophisticated technique, such as the full-wave Method of Moments (MoM), which is versatile and accurate, though highly computer-intensive.

In analyzing planar microstrip structures, the method of moments (MoM) can be applied either in the spectral domain [8]-[12], or in the spatial domain [13]-[17]. The spatial domain approach has the advantage that, in this method, the integrands for the MoM matrix elements need to be evaluated only over the finite support associated with the basis and testing functions, as opposed to over an infinite range required in its spectral domain counterpart [18]. However, in the conventional form of the spatial domain approach, the Green's functions for the microstrip structures involve the evaluation of the Sommerfeld integrals, whose integrands are highly oscillatory and slowly decaying functions; hence their

Manuscript received April 9, 1993; revised May 2, 1994.

I. Park and R. Mittra are with the Electromagnetic Communication Laboratory, Department of Electrical and Computer Engineering. University of Illinois at Urbana-Champaign, Urbana, IL 61801-2991 USA

M. Aksun is with the Department of Electrical and Electronic Engineering, Bilkent University, Ankara, Turkey.

IEEE Log Number 9407292. computation is very time consuming. However, it has recently been demonstrated in [19]-[21] that this problem can be obviated by using the newly-developed closed-form spatial domain Green's functions. The closed-form Green's function can be obtained by using a technique detailed in [22]. In this technique we extract the quasi-static images and the surface wave poles from the integrand of the Sommerfeld integral, and then handle their contributions analytically using the Sommerfeld identity and the residue theorem, respectively. Next, we approximate the remaining integrand in terms of a finite number of complex exponentials using the Generalized Pencil of Function (GPOF) [23] method. The objective of this paper is to employ these closed-form Green's functions to analyze general microstrip structures using the MoM approach.

The organization of the paper is as follows. Section II begins with the formulation of the problem in the context of MoM, and then goes on to present the scattering parameter analysis based upon the Generalized Eigenvalue Method. A number of microstrip discontinuities and patch antenna configurations, including patches with tuning stubs, are numerically analyzed in Section III, and the results are compared with those published previously in the literature.

\section{Formulation OF THE PROBlem}

The geometry of a general microstrip structure with a substrate and a superstrate is shown in Fig. 1. The substrate has a thickness of $d_{i-1}$ and a relative permittivity of $\varepsilon_{r i-1}$. The superstrate thickness is $d_{i}$ and its relative permittivity is $\varepsilon_{r i}$. The substrate, superstrate, and the ground plane are assumed to be infinitely wide in the horizontal plane, and the conductors are assumed to be lossless and infinitesimally thin. The time convention is $e^{j \omega t}$

The tangential components of the electric field on the plane of the patch can be written in terms of the surface current density, $\mathbf{J}$, and the Green's functions for the vector and scalar potentials, $G_{A}$ and $G_{q}$, respectively, as follows

$$
\begin{aligned}
& E_{x}=-j \omega G_{A}^{x x} * J_{x}+\frac{1}{j \omega} \frac{\partial}{\partial x}\left(G_{q} * \nabla \cdot \mathbf{J}\right) \\
& E_{y}=-j \omega G_{A}^{y y} * J_{y}+\frac{1}{j \omega} \frac{\partial}{\partial y}\left(G_{q} * \nabla \cdot \mathbf{J}\right)
\end{aligned}
$$

where $*$ denotes convolution. $G_{A}^{x \cdot x}$ represents the $x$-directed vector potential at $\mathbf{r}$ due to an $x$-directed electric dipole of unit strength located at $\mathbf{r}^{\prime}$, while $G_{q}$ represents the scalar potential 


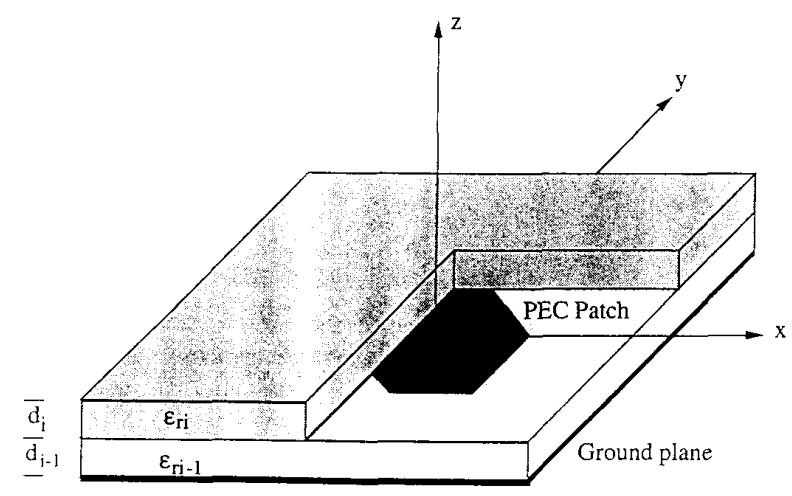

Fig. 1. A general microstrip structure with a substrate and a superstrate.

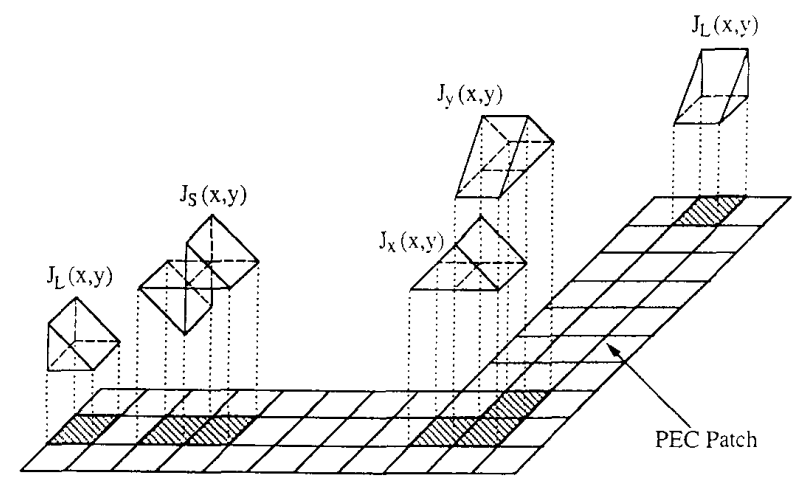

Fig. 2. Basis functions representing the current density on the microstrip patch.

produced by a unit point charge associated with a horizontal electric dipole (HED). The Green's functions appearing in (1) have algebraic singularities of the first order, i.e., $G \sim$ $O\left(1 /\left|\mathbf{r}-\mathbf{r}^{\prime}\right|\right)$. Hence, they are better suited for numerical computation than the Green's functions for the electric field integral equation which have algebraic singularities of the third order, viz., $O\left(1 /\left|\mathbf{r}-\mathbf{r}^{\prime}\right|^{3}\right)$.

\section{A. Application of the Method of Moments}

To solve for the surface current density on the patch using the MoM, the first step is to express the surface current density as a linear combination of the basis functions, which are chosen in this work to be rooftops (see Fig. 2). The $x$ - and $y$-components of the current density are expressed as:

$$
\begin{aligned}
& J_{x}(x, y)=\sum_{n} \sum_{m} I_{x}^{n m} \cdot J_{x}^{n m}(x, y)+J_{s}(x, y) \\
& J_{y}(x, y)=\sum_{n} \sum_{m} I_{y}^{n m} \cdot J_{y}^{n m} x, y
\end{aligned}
$$

where $J_{x}^{n m}$ and $J_{y}^{n m}$ are the rooftop functions, $J_{s}$ is the basis function for the current source, $I_{x}^{n m}$ and $I_{y}^{n m}$ are the unknown coefficients of the basis functions at the $(n . m)$ th position on the subdivided microstrip patch.

Substituting (2) into (1) and testing by applying the Galerkin's procedure, the matrix equations for the unknown coefficients of the basis functions can be obtained as

$$
\left[\begin{array}{ll}
Z_{x x}^{n^{\prime} m^{\prime}, n m} & Z_{x y}^{n^{\prime} m^{\prime}, n m} \\
Z_{y x}^{n n^{\prime} m^{\prime}, n m} & Z_{y y}^{n^{\prime} m^{\prime}, n m}
\end{array}\right]\left[\begin{array}{l}
I_{x}^{n m} \\
I_{y}^{n m}
\end{array}\right]=\left[\begin{array}{l}
V_{x}^{n^{\prime} m^{\prime}} \\
V_{y}^{n^{\prime} m^{\prime}}
\end{array}\right]
$$

where

$$
\begin{aligned}
Z_{x x}^{n^{\prime} m^{\prime}, n m}= & \left\langle\left\langle J_{x}^{n^{\prime} m^{\prime}}, G_{A}^{x x} * J_{x}^{n m}\right\rangle\right. \\
& \left.-\frac{1}{\omega^{2}}\left\langle\frac{\partial}{\partial x} J_{x}^{n^{\prime} m^{\prime}}, G_{q} * \frac{\partial}{\partial x} J_{x}^{n m}\right\rangle\right\} \\
Z_{x y}^{n^{\prime} m^{\prime}, n m}= & -\frac{1}{\omega^{2}}\left\langle\frac{\partial}{\partial x} J_{x}^{n^{\prime} m^{\prime}}, G_{q} * \frac{\partial}{\partial y} J_{y}^{n m}\right\rangle \\
Z_{y x}^{n^{\prime} m^{\prime}, n m}= & -\frac{1}{\omega^{2}}\left\langle\frac{\partial}{\partial y} \cdot J_{y}^{n^{\prime} m^{\prime}}, G_{q} * \frac{\partial}{\partial x} J_{x}^{n m}\right\rangle \\
Z_{y y}^{n^{\prime} m^{\prime}, n m}= & \left\langle\left\langle J_{y}^{n^{\prime} m^{\prime}} \cdot G_{A}^{y y} * J_{y}^{n m}\right\rangle\right. \\
& \left.-\frac{1}{\omega^{2}}\left\langle\frac{\partial}{\partial y} \cdot J_{y}^{n^{\prime} m^{\prime}}, G_{q} * \frac{\partial}{\partial y} \cdot J_{y}^{n m}\right\rangle\right\} \\
V_{x}^{n^{\prime} m^{\prime}}= & -\left\langle J_{x}^{n^{\prime} m^{\prime}} \cdot G_{A}^{x x} * J_{s}\right\rangle \\
& +\frac{1}{\omega^{2}}\left\langle\frac{\partial}{\partial x} J_{x}^{n^{\prime} m^{\prime}}, G_{q} * \frac{\partial}{\partial x} J_{s}\right\rangle \\
V_{y}^{n^{\prime} m^{\prime}}= & \frac{1}{\omega^{2}}\left\langle\frac{\partial}{\partial y} \cdot J_{y}^{n^{\prime} m^{\prime}} \cdot G_{q} * \frac{\partial}{\partial x} J_{s}\right\rangle .
\end{aligned}
$$

where $Z_{x x}^{n^{\prime} m^{\prime}, n m}$ denotes the mutual impedance between the $\left(n^{\prime}, m^{\prime}\right)$ th testing function and the $(n, m)$ th basis function, and $V_{x}^{n^{\prime} m^{\prime}}$ represents the excitation voltage at the $\left(n^{\prime}, m^{\prime}\right)$ th position of the element due to the current source.

Since the Green's functions appearing inside the inner product in (4) are available in closed-forms (See [18] for complete expressions), it is useful to transfer the convolution integrals involving the Green's functions and basis functions to the testing and basis functions instead, which can be chosen such that the integrals can be carried out analytically. This manipulation helps reduce the original fivefold integral to only a double integral, and results in a substantial savings in the computation time as a consequence.

The current densities at the load and source terminals, whenever they are used, are modeled by the half-rooftop basis functions. Although these basis functions have singularities in their derivatives, they do not present a problem and are handled according to the procedure given in [19]. The matrix equation in (4) can not be solved uniquely for the coefficients of the basis functions unless additional equations, obtained by imposing the boundary conditions at the load terminals, are added. They relate the coefficients of the load basis functions to the remainder of the basis functions in terms of the complex load impedances. For example, the additional equation at the left end of the load terminal can be written as (see [19]-[21])

$$
\left(1+j \beta_{x} h_{x} \frac{Z_{L 1}}{Z_{01}}-\frac{\beta_{x}^{2} h_{x}^{2}}{2}\right) I_{x}^{N\left(-M_{l}-1\right)}-I_{x}^{N\left(-M_{l}\right)}=0
$$

where $\beta_{x}$ and $Z_{01}$ are the propagation constant and the characteristic impedance of the line containing the load terminal, respectively.

By using these additional equations in the matrix (4), one can solve for the current distribution on the microstrip structure. 


\section{B. Scattering Parameter Analysis}

Once the current distributions on the microstrip structure have been found, the scattering parameters for general two port network can be computed by the following method. First, the line segments -1 and -2 , containing port- 1 and port-2, respectively, are modeled as transmission lines with characteristic impedances of $Z_{01}$ and $Z_{02}$. Next, Port- 1 is excited and the current distributions on segment- 1 and segment- 2 are computed. The transmission line is assumed to support only one propagating mode, since the reference plane is chosen to be sufficiently far away from the junction such that none of the higher-order modes are significant at the reference planes. For this model, the current distribution on segment- 1 can be expressed as

$$
\begin{aligned}
I_{11}(t) & =I_{11}^{+}(t)+I_{11}^{-}(t) \\
& =A_{11} e^{-j \beta_{1} t}+B_{11} e^{j \beta_{1} t}
\end{aligned}
$$

where $A_{11}$ and $B_{11}$ are the coefficients for the incident and reflected waves, respectively, and $\beta_{1}$ is propagation constant for the line segment-1. To determine the unknown coefficients, $A_{11}, B_{11}$, and $\beta_{1}$ in (6), we employ the Generalized Eigenvalue Method [23], and impose the constraint that the number of exponential terms representing the current distribution on the transmission line is only two, and that the two exponents are identical except for their sign difference. This procedure, outlined above, allows us to compute the propagation constant as well as the complex coefficients of the incident and reflected currents in the line segment-1.

Moving next to segment-2, we write the current distribution on it as

$$
\begin{aligned}
I_{21}(t) & =I_{21}^{+}(t)+I_{21}^{-}(t) \\
& =A_{21} e^{-j \beta_{2} t}+B_{21} e^{j \beta_{2} t}
\end{aligned}
$$

where $A_{21}$ and $B_{21}$ are the coefficients for the incident and reflected wave, respectively, and $\beta_{2}$ is propagation constant on segment- 2 . At the reference planes, $\left(A_{11}, B_{11}\right)$ and $\left(A_{21}\right.$, $\left.B_{21}\right)$ are equal to $\left(I_{11}^{+}, I_{11}^{-}\right)$, and $\left(I_{21}^{+}, I_{21}^{-}\right)$, respectively.

The four $S$-parameters, characterizing the two-port network, can be expressed as

$$
\left[\begin{array}{l}
S_{11} \\
S_{12} \\
S_{21} \\
S_{22}
\end{array}\right]=\left[\begin{array}{cccc}
I_{11}^{+} & I_{21}^{+} & 0 & 0 \\
0 & 0 & I_{11}^{+} & I_{21}^{+} \\
I_{12}^{+} & I_{22}^{+} & 0 & 0 \\
0 & 0 & I_{12}^{+} & I_{22}^{+}
\end{array}\right]^{-1}\left[\begin{array}{l}
I_{11}^{-} \\
I_{21}^{-} \\
I_{12}^{-} \\
I_{22}^{-}
\end{array}\right]
$$

which is the desired form we were seeking.

\section{RESUlTS AND Discussion}

In this section, we present some illustrative numerical results for three microstrip configurations: (i) an open-ended microstrip; (ii) microstrip line with a right-angle bend; and, (iii) microstrip line-fed patch antennas. The closed-form Green's functions used in this study are for general microstrip geometries with a substrate and a superstrate of arbitrary thicknesses. However, the dielectric constant of the superstrate is set to one so that our results can be compared with published results for the single layer cases.

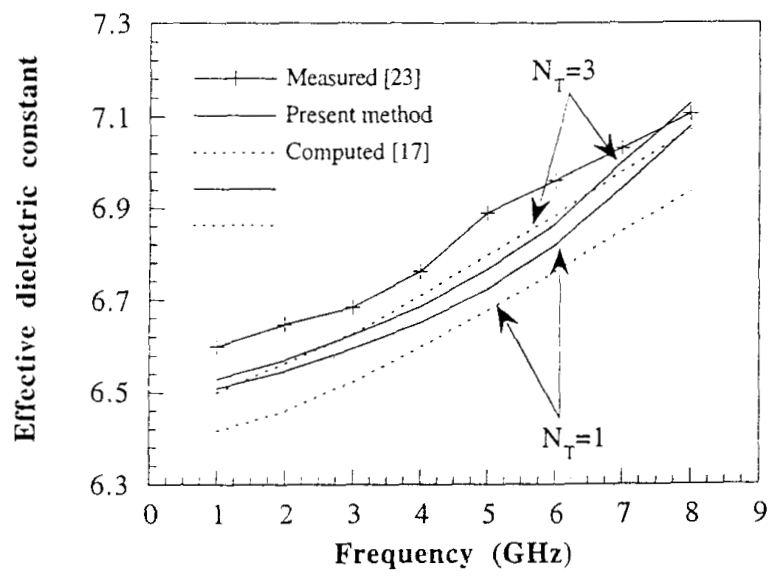

Fig. 3. Effective dielectric constant of an open microstrip line $\left(\varepsilon_{T i-1}=9.7\right.$, $d_{i-1}=1.27 \mathrm{~mm}, w=1.219 \mathrm{~mm}$ ).

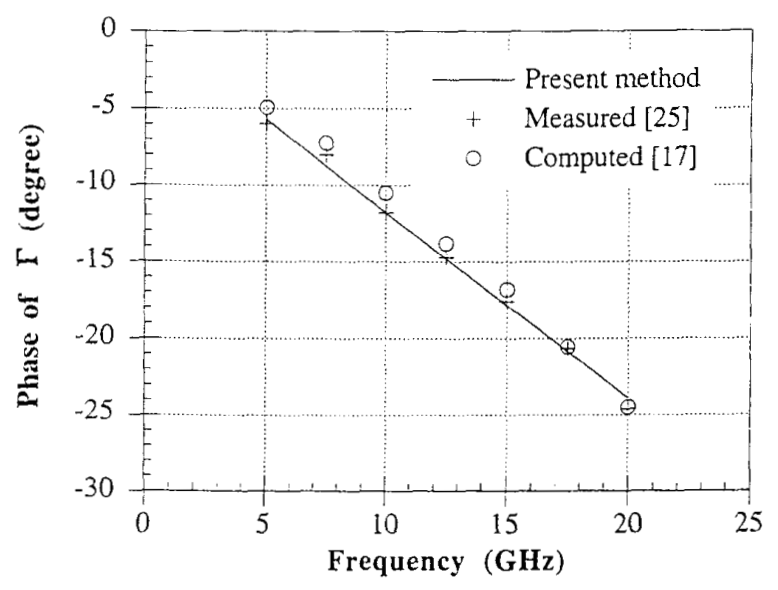

Fig. 4. Phase of the reflection coefficient of an open microstrip line $\left(\varepsilon_{r i-1}=9.9, d_{i-1}=0.635 \mathrm{~mm}, w=0.6033 \mathrm{~mm}\right)$.

\section{A. Microstrip Open-End}

As a first example that illustrates the accuracy of the method described in the last section, we consider the problem of modeling the discontinuity presented by the open end of a microstrip line. The following parameters are used for the computation: the dielectric constant and thickness of the substrate are $\varepsilon_{r i-1}=9.7$ and $d_{i-1}=1.27 \mathrm{~mm}$, respectively, and the width of the microstrip line is $w=1.219 \mathrm{~mm}$. The effective dielectric constants are computed and compared in Fig. 4 to the results given in [17] and with the measurement results from [24] (with graph reading errors of less than $0.2 \%$ ). In this computation, the half-wavelength long microstrip line was divided into 21 longitudinal segments. The computed results agree with those published in [17] to within $1 \%$, and with the measured data published in [24] to within $2 \%$ when the microstrip line is modeled with three transverse segments. Unlike in the procedure described in [17], the difference between the effective dielectric constants obtained by using one and three transverse segments is very small (less than $0.7 \%$ ) in the present method. This indicates that reasonably good results 


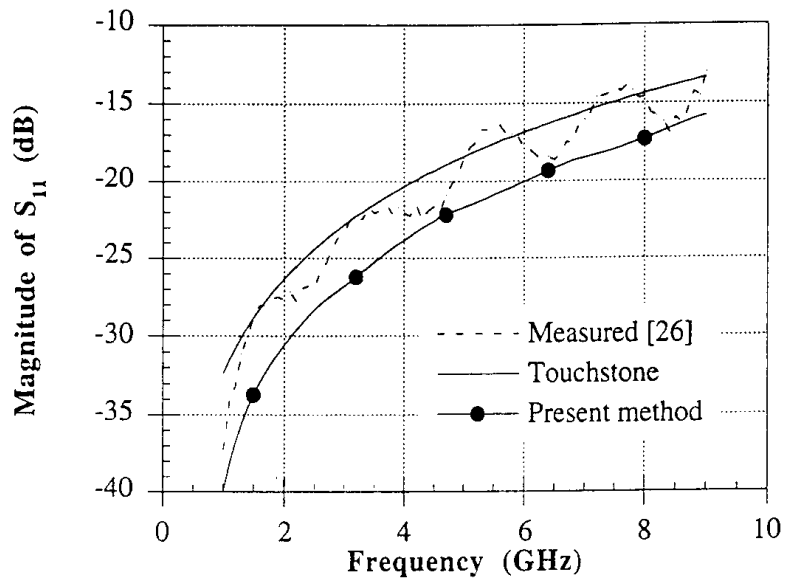

(a)

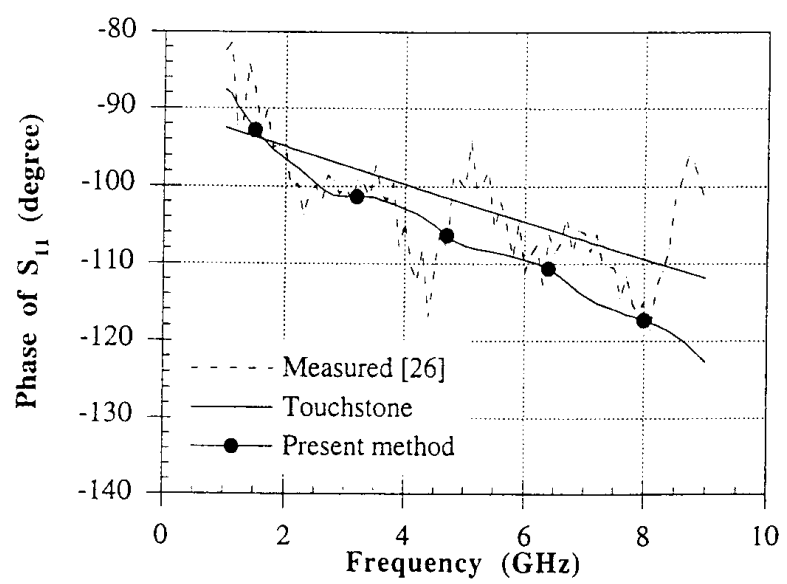

(b)

Fig. 5. Scattering parameter $S_{11}$ for the right-angled bend; (a) magnitude, (b) phase $\left(\varepsilon_{r i-1}=2.2, d_{i-1}=0.7874 \mathrm{~mm}, w=h x=2.4 \mathrm{~mm}\right)$.

can be obtained by using the procedure followed here without explicitly incorporating the edge condition, which, according to [25], requires approximately ten transverse segments for accurate modeling.

To complete the demonstration of the numerical accuracy of this method, the phase term of the reflection coefficient is computed for a microstrip line of $0.6033 \mathrm{~mm}$ width. The dielectric constant and thickness of the substrate are $\varepsilon_{r i-1}=$ 9.9 and $d_{i-1}=0.635 \mathrm{~mm}$, respectively. The numerical results obtained with the present method are compared in Fig. 5 to the computations presented in [17], and also to the measurements in [26], with graph reading errors of less than $0.2 \%$. For numerical computations, the half-wavelength long microstrip line is again divided into 21 longitudinal segments. The results obtained by using only one transverse segment are seen to compare very favorably with the measurements in $|17|$, as well as with the computed results given in [26].

\section{B. Microstrip Line With Right Angle Bend}

In the next example, we consider a microstrip discontinuity problem, viz., a right-angled bend. The dielectric constant of

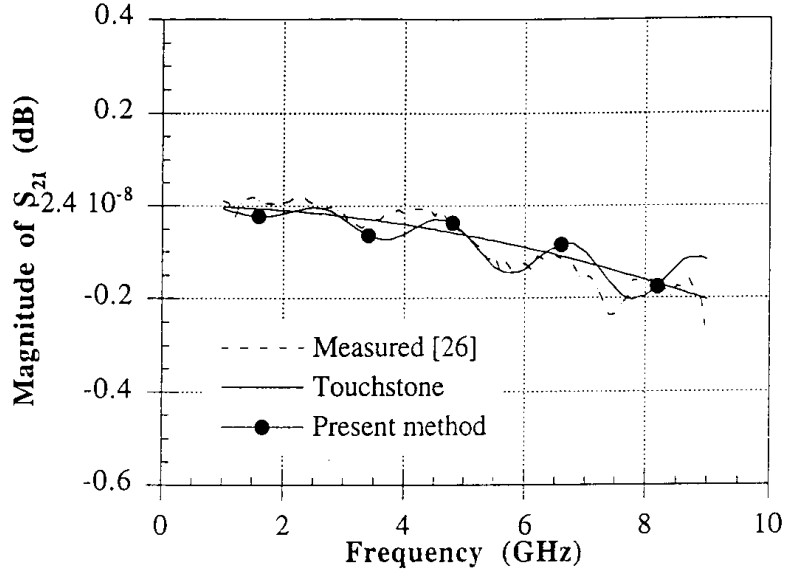

(a)

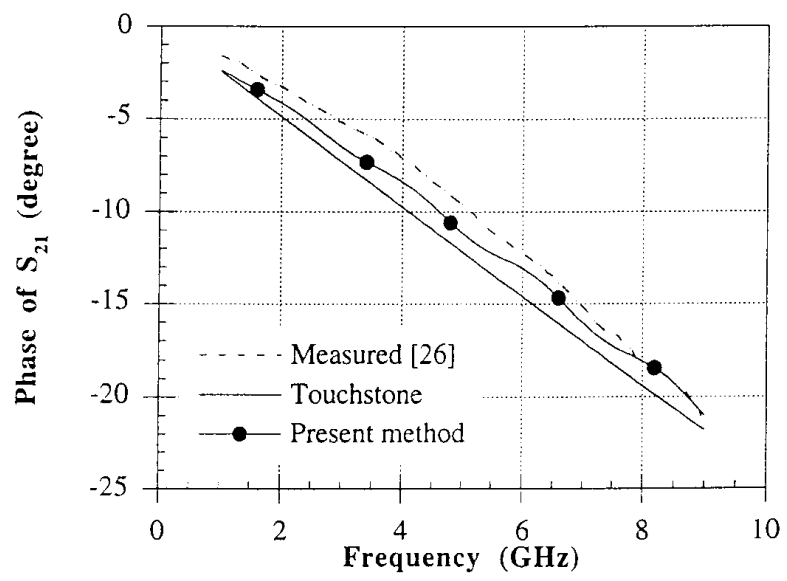

(b)

Fig. 6. Scattering parameter $S_{21}$ for the right-angled bend; (a) magnitude, (b) phase $\left(\varepsilon_{r i-1}=2.2, d_{i-1}=0.7874 \mathrm{~mm}, w=h x=2.4 \mathrm{~mm}\right)$.

the medium and the thickness of the substrate are chosen to be $\varepsilon_{r i-1}=2.2$ and $d_{i-1}=0.7874 \mathrm{~mm}$, respectively. The lengths of the segments are $L 1=L 2=55.2 \mathrm{~mm}$ and their widths are $w=h_{x}=2.4 \mathrm{~mm}$. The location of the current source is $4.8 \mathrm{~mm}$ from the left edge of segment- 1 . The computed and measured scattering parameters for the right-angled bend in a microstrip line are plotted in Figs. 5 and 6 as functions of frequency. The magnitude and phase of $S_{11}$ are compared with the quasi-static values and the experimental results of Harms [27], and are shown in Fig. 5. The results obtained with our method agree with the quasistatic values, as well as with the experimental data (within the measurement uncertainty) throughout the frequency range of comparison. Fig. 6 shows that the magnitude of the computed $S_{21}$ agrees with the measured data to within approximately $0.08 \mathrm{~dB}$, and its phase to within approximately 2 degrees of the measured values. The scattering parameters obtained by the present method have ripples since the characteristic impedances of the line are calculated using an empirical formula based on a quasi-static approach; these impedances, 

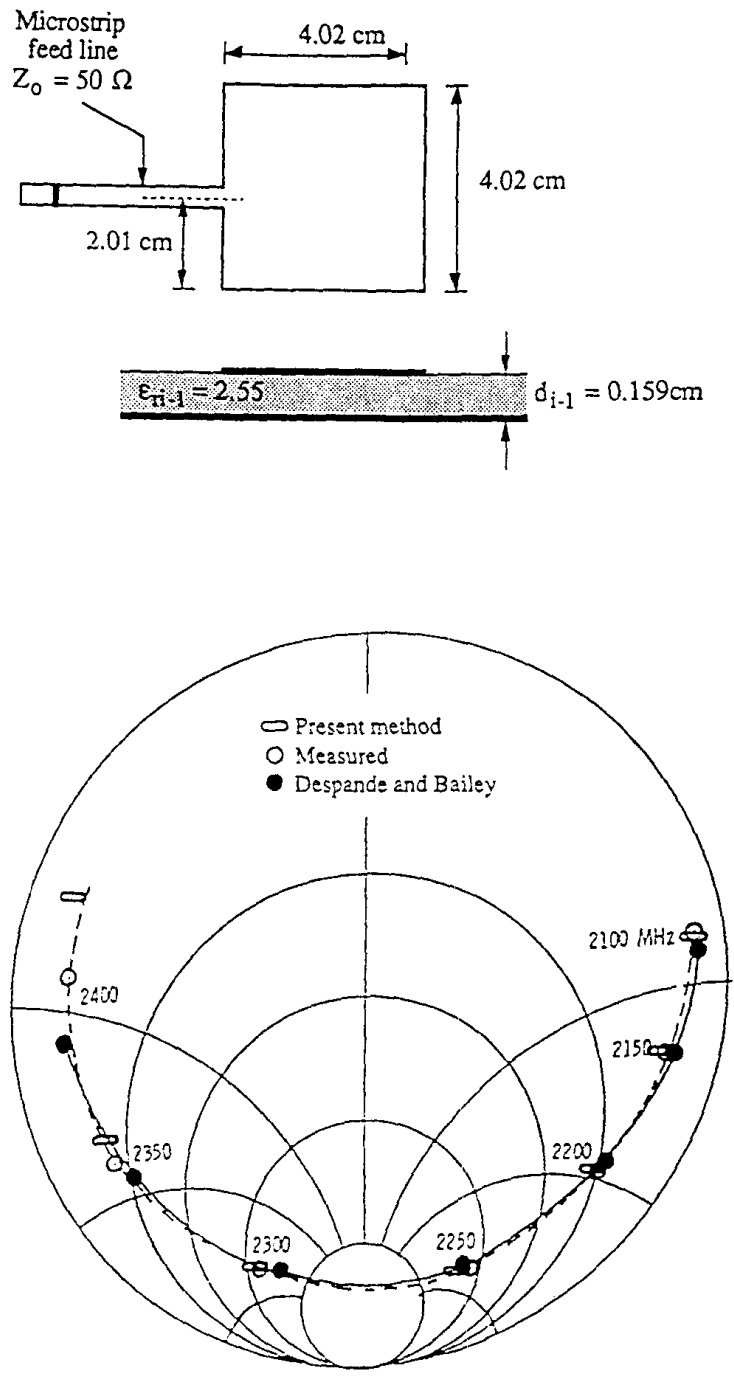

Fig. 7. Input impedance of a square microstrip antenna.

used as matched terminations at both ends, differ slightly from the true characteristic impedance of the line.

\section{Microstrip Line-Fed Patch Antenna}

Next, to illustrate the versatility of our method we consider a radiation type problem involving a microstrip patch antenna. The input impedance of a square patch fed by a microstrip line at the center of one edge is computed and compared with published results. In this example, the dielectric constant and thickness of the substrate are $\varepsilon_{r i-1}=2.55$ and $d_{i-1}=1.59$ $\mathrm{mm}$, respectively, the width $w$ of the feed line is $4.47 \mathrm{~mm}$ its length $L=116 \mathrm{~mm}$. The dimensions of the square patch are $a=b=40.2 \mathrm{~mm}$. The location of the source is 8.9 $\mathrm{mm}$ from the left edge of the feed line. The input impedance, computed using the present method, is compared in Fig. 7 with the experimental data given in Lo et al. [5], and the computed results of Deshpande and Bailey [9]. It is evident that the results obtained by using the present method are in excellent

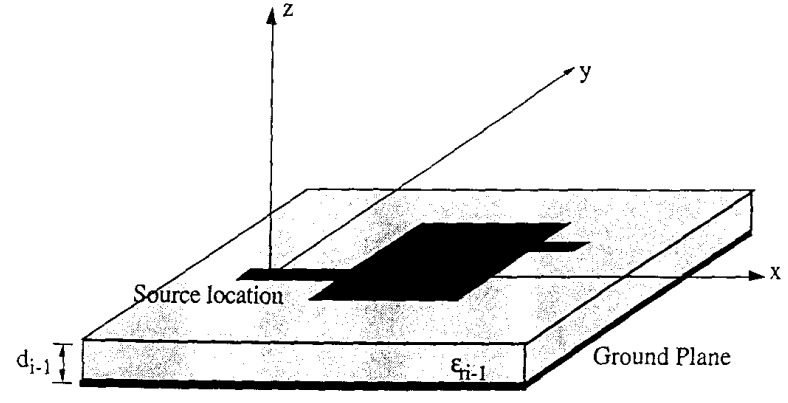

(a)

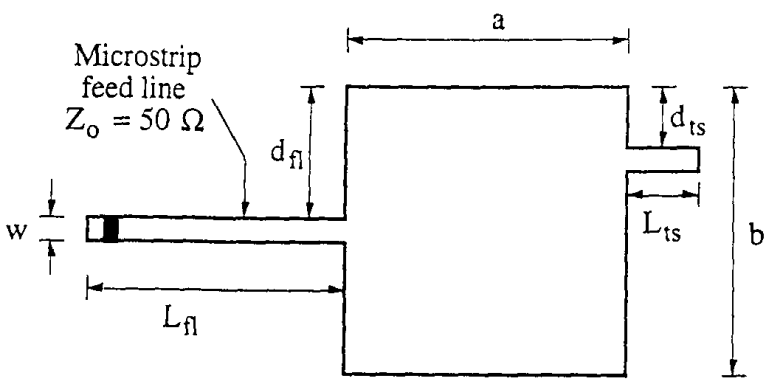

(b)

Fig. 8. (a) Geometry of a microstrip-line-fed patch antenna with a tuning stub, (b) top view of the geometry in (a).

agreement with the experimental, as well as other computed results over the frequency range of interest.

As a final example, we present the results of our investigation of a microstrip-line-fed patch antenna with a tuning stub, shown in Fig. 8. Both the feed line and the tuning stub are assumed to have open-ended terminations. These types of configurations are particularly useful [28] for fine-tuning the resonant frequency of a microstrip patch antenna by changing the length of the tuning stub, and/or its location along the radiating edges of the microstrip patch. In addition, patch antennas can be designed to radiate a circularly-polarized wave by making a judicious choice for the locations of the feed line and the tuning stub. The following parameters are used for the example given below, which illustrates the use of the tuning stub in microstrip patch antenna design. The dielectric constant of the medium and thickness of the substrate are $\varepsilon_{r i-1}=2.62$ and $d_{i-1}=0.794 \mathrm{~mm}$, respectively; the length $L_{\mathrm{fl}}$ of the feed line is $35.2 \mathrm{~mm}$ and its width $w_{\mathrm{f}}$ is $2.2 \mathrm{~mm}$. The width of the tuning stub is $2.2 \mathrm{~mm}$ and the dimensions of the square patch are $a=b=28.6 \mathrm{~mm}$. The location of the current source is $6.6 \mathrm{~mm}$ from the left edge of the feed line.

In the first study, the feed location is chosen at the center of left edge of the patch and the location of the tuning stub is moved from the top to the center of its right edge (see Fig. 8(b)). The magnitudes of the current distribution on the microstrip line-fed patch antenna, without and with the tuning stub, are shown in Figs. 9 and 10, respectively. As seen in Figs. 9(a) and 10(a), the $x$-components of the current distribution remain essentially unaffected by the presence of the tuning stub. However, Fig. 10(b) shows that the addition of the tuning stub induces a cross-polarization $(y)$ component 


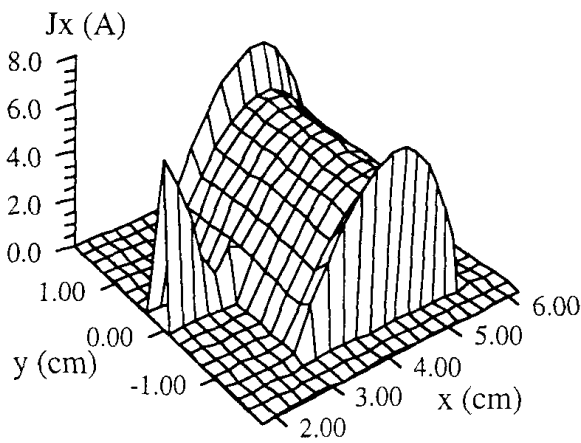

(a)

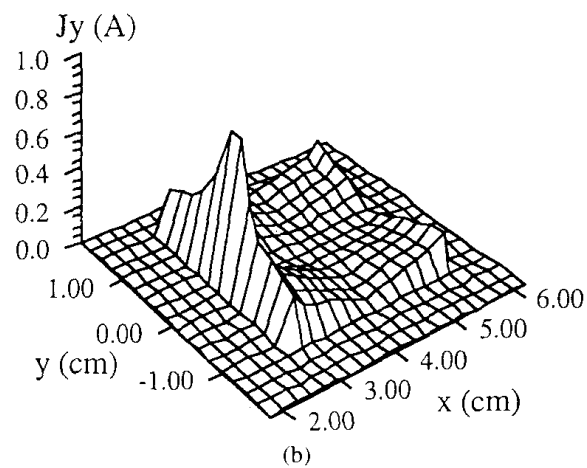

Fig. 9. Magnitudes of the current distribution on the microstrip line fed patch antenna (a) $J_{x}(x, y)$, (b) $J_{y}(x, y)$. Freq. $=3.16 \mathrm{GHz}, a=b=28.6 \mathrm{~mm}$, $D x=D y=2.2 \mathrm{~mm}, \varepsilon_{r i-1}=2.62, d_{i-1}=0.794 \mathrm{~mm}$.

in the patch current and excites a new mode along $y$. This leads us to conclude that both the $(1,0)$ and $(0,1)$ modes can be excited simultaneously using a tuning stub. It should now be evident that we can achieve circular polarization (CP) by adjusting the length and location of the tuning stub until the magnitudes of the $x$ - and $y$-components become equal and their relative phase shift becomes $90^{\circ}$. As is well known, the impedance locus exhibits a cusp-like behavior on the Smith Chart, as seen in Fig. 11, when the CP condition is achieved.

\section{CONCLUSION}

In this paper, spatial domain closed-form Green's functions have been employed for the analysis of a general class of microstrip structures. Numerical results for a uniform line, as well the scattering parameter analysis for a microstrip line with a right-angle bend have been found to agree well with experimental results as well as with those published elsewhere. The behavior of the input impedance of a microstrip-linefed patch antenna has been shown to agree closely with that computed by using the MoM approach in the spectral domain, which requires the computation of infinite integrals and is computer intensive. The analysis of the above patch antenna shows that the addition of a tuning stub is not only provides a convenient way to achieve fine tuning of the resonant frequency of the antenna, but is useful for achieving circularly-polarized radiation from the antenna as well.

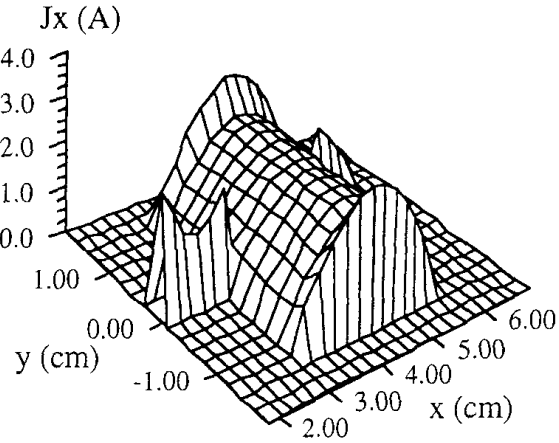

(a)

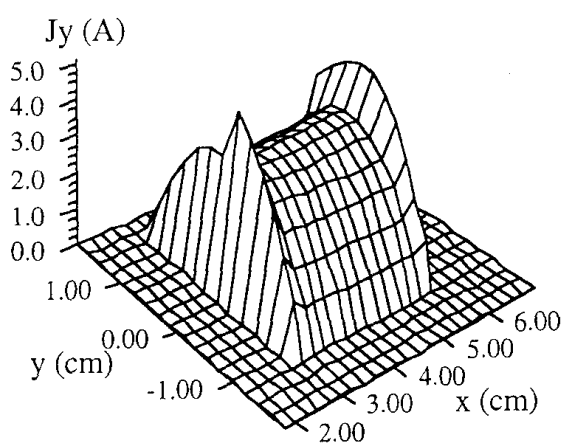

(b)

Fig. 10. Input impedance of the microstrip line center fed square patch antenna witha tuning stub. $f_{\text {start }}=2.98 \mathrm{GHz}, f_{\text {stop }}=3.30 \mathrm{GHz}$, $D f=0.01 \mathrm{GHz}$, dts $=4.4 \mathrm{~mm}$. (a) Lts $=2.2 \mathrm{~mm}$, (b) Lts $=4.4 \mathrm{~mm}$, (c) Lts $=6.6 \mathrm{~cm}$.

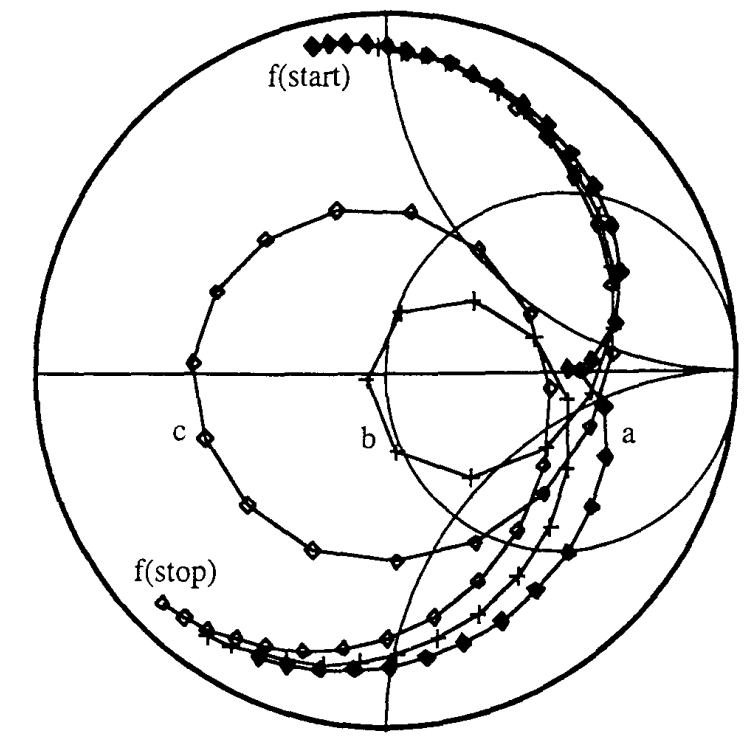

Fig. 11. Magnitudes of the current distribution on the microstrip line fed patch antenna with a tuning stub (a) $J_{x}(x, y)$, (b) $J_{y}(x, y)$. Freq $=3.16 \mathrm{GHz}$ $a=b=28.6 \mathrm{~mm}, D x=D y=2.2 \mathrm{~mm}, \Xi_{r-1}=2.62, d_{i-1}=0.794$ $\mathrm{mm}$, Lts $=8.8 \mathrm{~mm}$.

The use of the closed-form spatial domain Green's functions in the MoM formulation significantly reduces the computation time in comparison to that needed in the conventional 
formulation carried out in the spectral domain. For instance, in a numerical experiment with 40 roof-top basis functions, the CPU time for the solution of the current distribution was on the order of $1 \mathrm{~min}$. on a DEC station 5100 when the closed-form Green's functions were employed, whereas 100-150 mins. were required on the same workstation to solve the problem using the spectral domain moment method in conjunction with an acceleration technique.

We conclude that the method presented in this paper can be used to accurately solve for the current distributions on a variety of microstrip line geometries in less computation time than many other MoM approaches.

\section{REFERENCES}

[1] P. Silvester and P. Benedek, "Equivalent capacitance of microstrip open circuits," IEEE Trans. Microwave Theory Tech., vol. MTT-20, pp. 511-516, Aug. 1972.

[2] P. Benedek and P. Silvester, "Equivalent capacitance of microstrip gaps and steps," IEEE Trans. Microwave Theory Tech, vol. MTT-20, pp. 729-733, Nov. 1972.

[3] P. Silvester and P. Benedek, "Equivalent discontinuities capacitances for right-angle bends, T-junctions, and crossings," IEEE Trans. Microwave Theory Tech., vol. MTT-21, pp. 341-346, May 1973.

[4] T. Itoh, "Analysis of microstrip resonators," IEEE Trans. Microwave Theory Tech, vol. MTT-22, pp. 946-952, Nov. 1974.

[5] Y. T. Lo, D. Solomon, and W. F. Richads, "Theory and experiment on microstrip antennas," IEEE Trans. Antenn. Propagat,, vol. AP-27, pp. 137-145, Mar, 1979.

[6] R. Chandra and K. C. Gupta, "Segmentation method using impedance matrices for analysis of planar microwave circuits," IEEE Trans. Microwave Theory Tech, vol. MTT-29, pp. 71-74, Jan. 1981

[7] K. C. Gupta and P. C. Sharma, "Segmentation and desegmentation techniques for the analysis of planar microstrip antennas," presented at IEEE AP-S Int. Symp., Los Angeles, 1981.

[8] E. H. Newman and P. Tulyathan, "Analysis of microstrip antennas using moment methods," IEEE Trans. Antenn. Propagat., vol. AP-29, pp. $47-53,1981$

[9] M. D. Deshpande and M. C. Bailey, "Input impedance of microstrip antennas," IEEE Trans. Antenn. Propagat., vol. AP-30, pp. 645-650, 1982.

[10] D. M. Pozar, "Input impedance and mutual coupling of rectangular microstrip antennas," IEEE Trans. Antenn. Propagat, vol. AP-30, pp. 1191-1196, 1982

[11] R. W. Jackson and D. M. Pozar, "Full-wave analysis of microstrip openend and gap discontinuities," IEEE Trans. Microwave Theory Tech., vol. MTT-33, pp. 1036-1042, Oct. 1985.

[12] S. Wu, H. Yang, N. G. Alexopoulous, and I. Wolff, "A rigorous dispersive characterization of microstrip cross and $T$ junctions," IEE Trans. Microwave Theory Tech., vol. MTT-38, pp. 1837-1844, Dec. 1990.

[13] J. R. Mosig and F. E. Gardiol, "General integral equation formulation for microstrip antennas and scatterers," IEEE Proc., vol. 132, Pt. H, pp. 424-432, 1985.

[14] J. R. Mosig, "Arbitrarily shaped microstrip structures and their analysis with a mixed potential integral equation," IEEE Trans. Microwave Theory Tech., vol. MTT-36, pp. 314-323, Feb. 1988.

[15] W. P. Harokopus and P. B. Katehi, "Characterization of microstrip discontinuities on multilayer dielectric substrates including radiation losses," IEEE Trans. Microwave Theory Tech., vol. MTT-37, pp. 2058-2065, 1989

[16] K. A. Michalski and D. Zheng, "Analysis of microstrip resonators of arbitrary shape," IEEE Trans. Microwave Theory Tech., vol. MTT-40, pp. 112-119, Jan. 1992 .

[17] F. Alonso-Monferrer, A. A. Kishk, and A. W. Glisson, "Green's functions analysis of planar circuits in a two-layer grounded medium," IEEE Trans. Antenn. Propagat., vol. AP-40, pp. 690-696, June 1992.

[18] M. I. Aksun and R. Mittra, "Derivation of closed form Green's functions for a general microstrip geometry," IEEE Trans. Microwave Theory Tech., vol. MTT-40, pp. 2055-2062, 1992.

[19] "Estimation of spurious radiation from microstrip etches using closed-form Green's function," IEEE Trans. Microwave Theory Tech., vol. MTT-40, pp. 2063-2069, 1992.
[20] I. Park and R. Mittra, "Efficient computations for a general microstrip geometry using closed-form spatial domain Green's functions," Electromagnetic Communication Lab., Dept. of Elect. and Comp. Eng., Univ. of Illinois, Tech. Rep. No. 93 , Feb. 1993.

[21] M. I. Aksun and R. Mittra, "Spurious radiation from microstrip interconnects," IEEE Trans. Electromagn. Compat., vol. 35, pp. 148-158, 1993.

[22] Y. L. Chow, J. J. Yang, D. H. Fang, and G. E. Howard, "Closed-form spatial Greens function for the thick substrate," IEEE Trans. Microwave Theory Tech., vol. MTT-39, pp. 588-592, 1991.

[23] T. K. Sarkar, Z. A. Maricevic, and M. Kahrizi, "An accurate deembedding procedure for characterizing discontinuities," $I n t^{\prime} l . J$ Microwave and Millimeter-Wave Computer-Aided Eng., vol. 2., pp. 135-143, 1992.

[24] C. P. Hartwig, D. Masse, and R. A. Pucel, "Frequency dependent behavior of microstrip," in IEEE Group on Microwave Theory Tech. Microwave Symp. Dig., pp. 110-116, 1968

[25] E. Michielssen, Private communication.

[26] G. Gronau and I. Wolff, "A simple broad-band device de-embedding method using automatic network analyzer with time-domain option," IEEE Trans. Microwave Theory Tech., vol. 37, pp. 479-483, Mar. 1989

[27] P. Harms, "Numerical modeling of microstrip discontinuities and related structures," Ph.D. dissertation, Dept. of Elect. and Comp. Eng., Univ. of Illinois, Urbana, IL, 1992.

[28] K. R. Carver and J. W. James, "Microstrip antenna technology," IEEE Trans. Antenn. Propagat., vol. AP-29, pp. 2-24, Jan. 1981

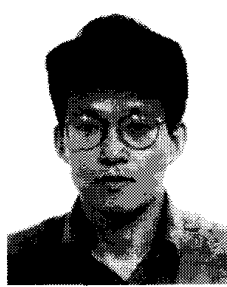

Ikmo Park received the B.E. Degree in Electrical Engineering from the State University of New York at Stony Brook in 1984, and the M.S. and Ph.D degrees in Electrical Engineering from the University of Illinois at Urbana-Champaign in 1989 and 1994 , respectively.

He has been employed by Human Devices, Inc. from 1986 to 1987 . He is currently a post-doctoral Fellow in the Electromagnetic Communication Laboratory in the Departrnent of Electrical and Computer Engineering at the University of Illinois.

Dr. Park present research interests include numerical methods for electromagnetics, microstrip antennas and electronic packing for high-speed digital interconnections. He is a member of Eta Kappa Nu and Tau Beta Pi.

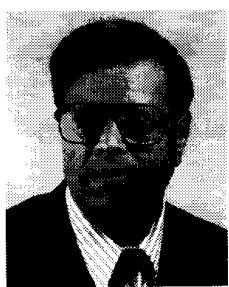

Raj Mittra (S'54-M'57-SM'69-F'71) is the Director of the Electromagnetic Communication Laboratory of the Electrical and Computer Engineering Department and Research Professor of the Coordinated Science Laboratory at the University of Illinois

He has served as the editor of the Transactions of the Antennas and Propagation Society. He has been a Visiting Professor at Oxford University, Oxford, England and at the Technical University of Denmark, Lyngby, Denmark. Currently, he serves as the North American editor of the journal AEU. He is President of RM Associates, which is a consulting organization providing services to several industrial and governmental organizations. His professional interests include the areas of electromagnetic modeling and simulation of electronic packages, EMP and EMC analysis, radar scattering, frequency selective surfaces, microwave and millimeter wave integrated circuits, and satellite antennas. He has published over 350 journal papers and 20 books or book chapters on various topics related to electromagnetics.

Dr Mittra is a Fellow of the IEEE, a Past-President of AP-S, He won the Guggenheim Fellowship Award in 1965 and the IEEE Centennial Medal in 1984.

M. I. Aksun, photograph and biography not available at time of publication 